\title{
Classification of Hyperspectral and LiDAR Data Using Coupled CNNs
}

\author{
Renlong Hang ${ }^{\circledR}$, Member, IEEE, Zhu Li, Senior Member, IEEE, Pedram Ghamisi ${ }^{\circledR}$, Senior Member, IEEE, \\ Danfeng Hong ${ }^{\circledR}$, Member, IEEE, Guiyu Xia ${ }^{\circledR}$, and Qingshan Liu ${ }^{\circledR}$, Senior Member, IEEE
}

\begin{abstract}
In this article, we propose an efficient and effective framework to fuse hyperspectral and light detection and ranging (LiDAR) data using two coupled convolutional neural networks (CNNs). One CNN is designed to learn spectral-spatial features from hyperspectral data, and the other one is used to capture the elevation information from LiDAR data. Both of them consist of three convolutional layers, and the last two convolutional layers are coupled together via a parameter-sharing strategy. In the fusion phase, feature-level and decision-level fusion methods are simultaneously used to integrate these heterogeneous features sufficiently. For the feature-level fusion, three different fusion strategies are evaluated, including the concatenation strategy, the maximization strategy, and the summation strategy. For the decision-level fusion, a weighted summation strategy is adopted, where the weights are determined by the classification accuracy of each output. The proposed model is evaluated on an urban data set acquired over Houston, USA, and a rural one captured over Trento, Italy. On the Houston data, our model can achieve a new record overall accuracy $(\mathrm{OA})$ of $96.03 \%$. On the Trento data, it achieves an $\mathrm{OA}$ of $\mathbf{9 9 . 1 2 \%}$. These results sufficiently certify the effectiveness of our proposed model.
\end{abstract}

Index Terms-Convolutional neural networks (CNNs), decision fusion, feature fusion, hyperspectral data, light detection and ranging (LiDAR) data, parameter sharing.

Manuscript received September 15, 2019; revised November 20, 2019; accepted January 14, 2020. Date of publication February 6, 2020; date of current version June 24, 2020. This work was supported in part by the Natural Science Foundation of United States under Grant 1747751, in part by the Natural Science Foundation of China under Grant 61825601, Grant 61532009, Grant 61906096, and Grant 61802198, and in part by the Natural Science Foundation of Jiangsu Province, China, under Grant BK20180786, Grant BK20180788, and Grant 18KJB520032. (Corresponding author: Zhu Li.)

Renlong Hang is with the Jiangsu Key Laboratory of Big Data Analysis Technology, School of Automation, Nanjing University of Information Science and Technology, Nanjing 210044, China, and also with the Department of Computer Science and Electrical Engineering, University of Missouri-Kansas City, Kansas City, MO 64110 USA (e-mail: renlong_hang@163.com).

Zhu Li is with the Department of Computer Science and Electrical Engineering, University of Missouri-Kansas City, Kansas City, MO 64110 USA (e-mail: lizhu@umkc.edu).

Pedram Ghamisi is with the Helmholtz-Zentrum Dresden-Rossendorf (HZDR), Helmholtz Institute Freiberg for Resource Technology (HIF), D-09599 Freiberg, Germany (e-mail: p.ghamisi@gmail.com).

Danfeng Hong is with the Remote Sensing Technology Institute (IMF), German Aerospace Center (DLR), 82234 Wessling, Germany, and also with Signal Processing in Earth Observation (SiPEO), Technical University of Munich (TUM), 80333 Munich, Germany (e-mail: danfeng.hong@dlr.de).

Guiyu Xia and Qingshan Liu are with the Jiangsu Key Laboratory of Big Data Analysis Technology, School of Automation, Nanjing University of Information Science and Technology, Nanjing 210044, China (e-mail: xiaguiyu1989@sina.com; qsliu@nuist.edu.cn).

Color versions of one or more of the figures in this article are available online at http://ieeexplore.ieee.org.

Digital Object Identifier 10.1109/TGRS.2020.2969024

\section{INTRODUCTION}

A CCURATE land-use and land-cover classification plays an important role in many applications such as urban planning and change detection. In the past few years, hyperspectral data have been widely explored for this task [1]-[3]. Compared to multispectral data, hyperspectral data have more rich spectral information, ranging from the visible spectrum to the infrared spectrum [4]. Such information, combined with some spatial information in hyperspectral data, can generally acquire satisfying classification results [5], [6]. However, for urban and rural areas, there often exist many complex objects that are difficult to discriminate because they have similar spectral responses. Thanks to the development of remote sensing technologies, nowadays, it is possible to measure different aspects of the same object on the Earth's surface [7]. Different from hyperspectral data, light detection and ranging (LiDAR) data can record the elevation information of objects, thus providing complementary information for the hyperspectral data. For instance, if both the building roof and the road are made up of concrete, it is very difficult to distinguish them using only hyperspectral data since their spectral responses are similar. However, LiDAR data can accurately classify those two classes as they have different heights. On the contrary, LiDAR data cannot differentiate between two different roads, which are made up of different materials (e.g., asphalt and concrete), having the same height. Therefore, fusing hyperspectral and LiDAR data is a promising scheme whose performance has already been validated in the literature for land-cover and land-use classification [7], [8].

In order to take advantage of the complementary information between hyperspectral and LiDAR data, a lot of works have been proposed. One widely used class of methods is based on feature-level fusion. In [9], morphological extended attribute profiles (EAPs) were applied to hyperspectral and LiDAR data. These profiles and the original spectral information of hyperspectral data were stacked together for classification. However, the direct stacking of these highdimensional features inevitably results in the well-known Hughes phenomenon, especially when only a relatively small number of training samples is available. To address this issue, principal component analysis (PCA) was employed to reduce the dimensionality. Similar to this article, many subspacerelated models can be designed to fuse the extracted spectral, spatial, and elevation features [10]-[14]. For example, a graph 
embedding framework was proposed by Liao et al. [11]; a low-rank component analysis model was proposed by Rasti et al. [12]. Different from them, Gu et al. [16] attempted to use multiple-kernel learning [15] to combine heterogeneous features. They constructed a kernel for each feature and then combined these kernels together in a weighted summation manner. Different weights can represent the importance of different features for classification.

Besides the feature-level fusion, decision-level fusion is another popularly adopted method. In [17], spectral features, spatial features, elevation features, and their fused features were fed into the support vector machine (SVM) individually to generate four classifiers, and the final classification result was determined by them. In [18], two different fusion strategies named hard decision fusion and soft decision fusion were used to integrate the classification results from a different data source. Their fusion weights were uniformly distributed. In [19], three different classifiers, including the maximum likelihood classifier, SVM, and the multinomial logistic regression, were used to classify the extracted features. The fusion weights for these classifiers were adaptively optimized by a differential evolution algorithm. Recently, a novel ensemble classifier using random forest was proposed, in which a majority voting method was used to produce the final classification result [20]. In summary, the difference between feature-level fusion and decision-level fusion methods lies in the phase where the fusion process happens, but both of them require powerful representations of hyperspectral and LiDAR data. To achieve this goal, one needs to spend a lot of time designing appropriate feature extraction and feature selection methods. These handcrafted features often require domain expertise and prior knowledge.

In recent years, deep learning has attracted more and more attention in the field of remote sensing [21], [22]. In contrast to the handcrafted features, deep learning can learn high-level semantic features from data itself in an end-to-end manner [23]. Among various deep learning models, convolutional neural networks (CNNs) gain the most attention and have been explored in various tasks. For example, in [24], CNN was applied to object detection in remote sensing images. In [25], three CNN frameworks were proposed for hyperspectral image classification. Liu et al. [26] used CNNs to learn multiscale deep features for remote sensing image scene classification. Due to its powerful feature learning ability, some researchers attempted to use CNN for hyperspectral and LiDAR data fusion recently. An early attempt appears in [27]. It directly considered LiDAR data as another spectral band of hyperspectral data, and then fed the concatenated data into CNN to learn features and perform classification. Ghamisi et al. [28] tried to combine the traditional feature extraction method and CNN together. They fed the fused features to $\mathrm{CNN}$ for learning a higher-level representation and getting a classification result. Similarly, Li et al. [29] constructed three CNNs to learn spectral, spatial, and elevation features, respectively, and then used a composite kernel method to fuse them. Different from them, an end-to-end CNN fusion model was designed in [30], which embedded feature extraction, feature fusion, and classification into one framework. Specifically, the hyperspectral and LiDAR data were directly fed into their corresponding CNNs to extract features, and then these features were concatenated together, followed by a fully connected layer to further fuse them. Based on this two-branch framework, Xu et al. [31] also proposed a spectral-spatial CNN for hyperspectral data analysis and another spatial CNN for LiDAR data analysis.

It is well-known that the performance of CNN-based models heavily depends on the number of available samples. However, in the field of hyperspectral and LiDAR data fusion, there often exists a small number of training samples. To address this issue, an unsupervised CNN model was proposed in [32] based on the famous encoder-decoder architecture [33]. Specifically, it first mapped the hyperspectral data into a hidden space via an encoding path, and then reconstructed the LiDAR data with a decoding path. After that, the hidden representation in the encoding path can be considered as fused features of hyperspectral and LiDAR data. Nevertheless, there still exist some issues. For example, the loss of supervised information from labeled samples will lead to a suboptimal feature representation; it also needs to design another network to classify the learned representation, which will increase the computation complexity. In this article, we propose a supervised model to fuse hyperspectral and LiDAR data by designing an efficient and effective CNN framework. Similar to [30], we also use two CNNs but with a more efficient representation. We use three convolutional layers with small kernels (i.e., $3 \times 3$ ), and two of them share parameters. Besides the output layer, we do not use any fully connected layers. The major contributions of this article are summarized as follows.

1) In order to sufficiently fuse hyperspectral and LiDAR data, two coupled CNNs are designed. Compared to the existing CNN-based fusion models, our model is more efficient and effective. The coupled convolution layers can reduce the number of parameters, and more importantly, guide the two CNNs learn from each other, thus facilitating the following feature fusion process.

2) In the fusion phase, we simultaneously use feature-level and decision-level fusion strategies. For the feature-level fusion, we propose summation and maximization fusion methods in addition to the widely adopted concatenation method. To enhance the discriminative ability of learned features, we add two output layers to the CNNs, respectively. These three output results are finally combined together via a weighted summation method, whose weights are determined by the classification accuracy of each output on the training data.

3) We test the effectiveness of the proposed model on two data sets using standard training and test sets. On the Houston data, we can achieve an overall accuracy (OA) of $96.03 \%$, which is the best result ever reported in the literature. On the Trento data, we can also obtain very high performance (i.e., an OA of $99.12 \%$ ).

The rest of this article is organized as follows. Section II describes the details of the proposed model, including the coupled CNN framework, the data fusion model, and the network training and testing methods. The descriptions of data 


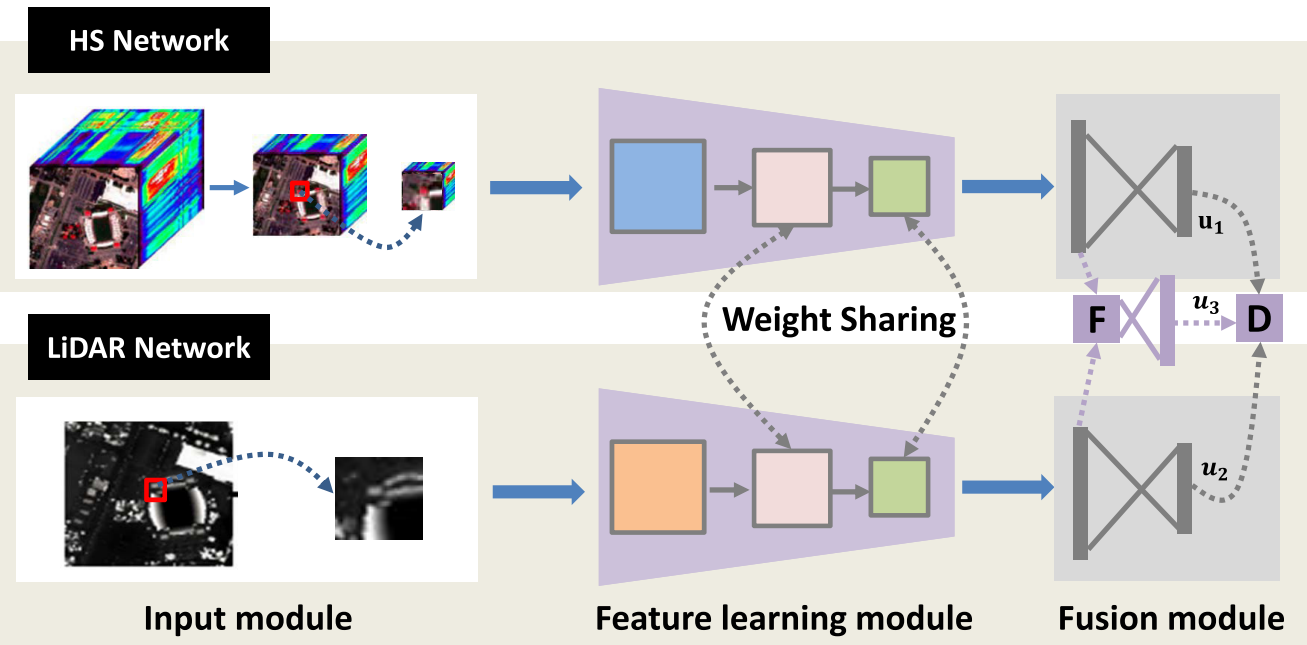

Fig. 1. Flowchart of the proposed model.

sets and experimental results are given in Section III. Finally, Section IV concludes this article.

\section{Methodology}

\section{A. Framework of the Proposed Model}

As shown in Fig. 1, our proposed model mainly consists of two networks: an HS network for spectral-spatial feature learning and a LiDAR network for elevation feature learning. Each of them includes an input module, a feature learning module, and a fusion module. For the HS network, PCA is firstly used to reduce the redundant information of the original hyperspectral data, and then a small cube is extracted surrounding the given pixel. For the LiDAR network, we can directly extract an image patch at the same spatial position as the hyperspectral data. In the feature learning module, we use three convolutional layers, and the last two of them share parameters. In the fusion module, we construct three classifiers. Each CNN has an output layer, and their fused features are also fed into an output layer.

\section{B. Feature Learning via Coupled CNNs}

Given a hyperspectral image $\mathbf{X}_{h} \in \mathfrak{R}^{m \times n \times b}$ and a corresponding LiDAR image $\mathbf{X}_{l} \in \mathfrak{R}^{m \times n}$ covering the same area on the Earth's surface. Here, $m$ and $n$ represent the height and width, respectively, of the two images, and $b$ refers to the number of spectral bands of the hyperspectral image. Our goal is to sufficiently fuse the information from $\mathbf{X}_{h}$ and $\mathbf{X}_{l}$ to improve the classification performance. As with any other classification tasks, feature representation is a critical step here. Due to the effects of multipath scattering and the heterogeneity of subpixel constituents, $\mathbf{X}_{h}$ often exhibits nonlinear relationships between the captured spectral information and the corresponding material. This nonlinear characteristic will be magnified when dealing with $\mathbf{X}_{l}$ [7]. It has been proved that CNNs are capable of extracting high-level features, which are usually invariant to the nonlinearities of hyperspectral [34]-[36] and LiDAR data [30], [37]. Inspired from them,

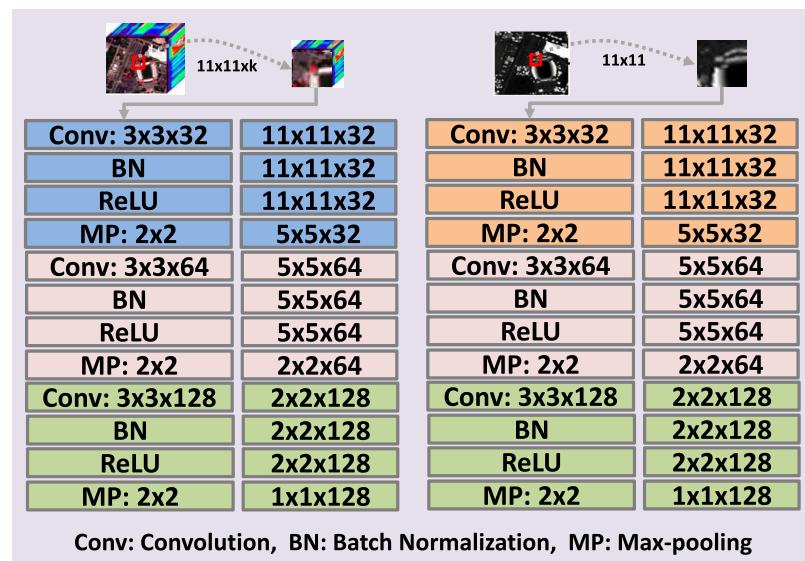

Fig. 2. Architecture of the coupled CNNs.

we design a coupled CNN framework to learn features from $\mathbf{X}_{h}$ and $\mathbf{X}_{l}$ efficiently.

The detailed architecture of the coupled CNNs is demonstrated in Fig. 2. First of all, PCA is used to extract the first $k$ principle components of $\mathbf{X}_{h}$ to reduce the redundant spectral information. Then, for each pixel, a small cube $\boldsymbol{x}_{h} \in \mathfrak{R}^{p \times p \times k}$ and a small patch $\boldsymbol{x}_{l} \in \mathfrak{R}^{p \times p}$ centered at it are chosen from $\mathbf{X}_{h}$ and $\mathbf{X}_{l}$, respectively. According to [30] and [32], the neighboring size $p$ can be empirically set to 11 . After that, $\boldsymbol{x}_{h}$ and $\boldsymbol{x}_{l}$ are fed into three convolutional layers to learn features. For the first convolutional layer, we adopt two different convolution operators (the blue box and the orange box) to obtain an initial representation of $\boldsymbol{x}_{h}$ and $\boldsymbol{x}_{l}$, respectively. This convolutional layer is sequentially followed by a batch normalization $(\mathrm{BN})$ layer to regularize and accelerate the training process, a rectified linear unit (ReLU) to learn a nonlinear representation, and a max-pooling layer to reduce the data variance and the computation complexity.

For the second convolutional layer, we let the HS network and the LiDAR network share parameters. Such a coupling strategy has at least two benefits. First, it can significantly 


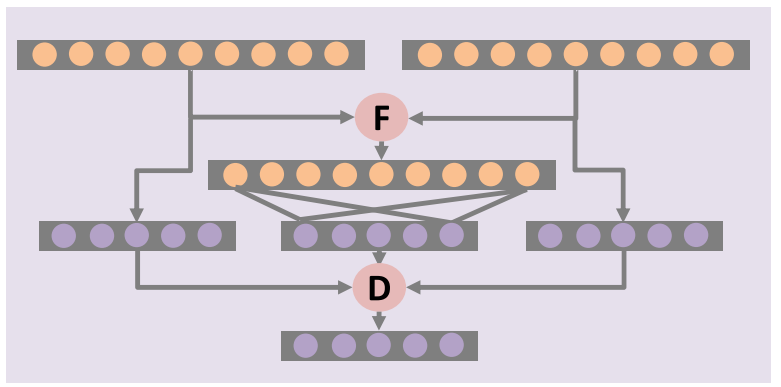

Fig. 3. Structure of the fusion module.

reduce the number of parameters twice, which is very useful with a small number of training samples. Second, it can make these two networks learn from each other. Without weight sharing, the training parameters in each network will be optimized independently using their own loss functions. After adopting the coupling strategy, the backpropagated gradients to this layer will be determined by the loss functions of both networks, which means that the information in one network will directly affect the other one. For the third convolutional layer, we also use the coupling strategy, which can further improve the discriminative ability of the learned representation from the second convolutional layer. Again, these two convolutional layers are followed by BN, ReLU, and max-pooling operators. The sizes (i.e., $3 \times 3$ ) and the number of kernels (i.e., 32, 64, and 128 sequentially) of each convolutional layer are shown at the left side under each data. Similarly, the output size (e.g., $11 \times 11 \times 32$ ) of each operator is shown at the right side. It is worth noting that all the convolutional layers have padding operators to make the output size the same as the input size.

\section{Hyperspectral and LiDAR Data Fusion}

After getting the feature representations of $\boldsymbol{x}_{h}$ and $\boldsymbol{x}_{l}$, how to combine them becomes another important issue. Most of the existing deep learning models [30]-[32] choose to stack them together and use a few fully connected layers to fuse them. However, fully connected layers often contain a large number of parameters, which will increase the training difficulty when there exists only a small number of training samples. To this end, we propose a novel combination strategy based on feature-level and decision-level fusions. Assume $\mathbf{R}_{h} \in \mathfrak{R}^{128 \times 1}$ and $\mathbf{R}_{l} \in \mathfrak{R}^{128 \times 1}$ denote the learned features for $\boldsymbol{x}_{h}$ and $\boldsymbol{x}_{l}$, respectively. As shown in Fig. 3, we first combine $\mathbf{R}_{h}$ and $\mathbf{R}_{l}$ to generate a new feature representation. Then, we input these three features into output layers separately. Finally, all the output layers are integrated together to produce a final result. The whole fusion process can be formulated as

$$
\mathbf{O}=D\left[f_{1}\left(\mathbf{R}_{h} ; \mathbf{W}_{1}\right), f_{2}\left(\mathbf{R}_{l} ; \mathbf{W}_{2}\right), f_{3}\left(F\left(\mathbf{R}_{h}, \mathbf{R}_{l}\right) ; \mathbf{W}_{3}\right) ; \mathbf{U}\right]
$$

where $\mathbf{O} \in \mathfrak{R}^{C \times 1}$, where $C$ is the number of classes to discriminate, represents the final output of the fusion module; $D$ and $F$ are decision-level and feature-level fusions, respectively; $f_{1}, f_{2}$, and $f_{3}$ are three output layers connected to $\mathbf{R}_{h}, \mathbf{R}_{l}$, and $F\left(\mathbf{R}_{h}, \mathbf{R}_{l}\right)$, respectively; $\mathbf{W}_{1} \in \mathfrak{R}^{C \times 128}, \mathbf{W}_{2} \in$
$\mathfrak{R}^{C \times 128}, \mathbf{W}_{3} \in \mathfrak{R}^{C \times 128}$, denote the connection weights for $f_{1}, f_{2}$, and $f_{3}$, respectively; $\mathbf{U} \in \mathfrak{R}^{C \times 3}$ corresponds to the fusion weight for $D$.

For the feature-level fusion $F$, we use summation and maximization methods in addition to the widely used concatenation method. The summation fusion aims to compute the sum of the two representations

$$
F\left(\mathbf{R}_{h}, \mathbf{R}_{l}\right)=\mathbf{R}_{h}+\mathbf{R}_{l} .
$$

Similarly, the maximization fusion aims at performing an element-wise maximization

$$
F\left(\mathbf{R}_{h}, \mathbf{R}_{l}\right)=\max \left(\mathbf{R}_{h}, \mathbf{R}_{l}\right) .
$$

Obviously, the performance of $F$ depends on its inputs $\mathbf{R}_{h}$ and $\mathbf{R}_{l}$. Therefore, we add two output layers $f_{1}$, and $f_{2}$ to supervise their learning processes. In the output phase, they can also help make decisions. The output value of $f_{1}$ can be derived as follows:

$$
\hat{\mathbf{y}}_{1}=f_{1}\left(\mathbf{R}_{h} ; \mathbf{W}_{1}\right)=\operatorname{softmax}\left(\mathbf{W}_{1} \mathbf{R}_{h}\right)
$$

where softmax represents the softmax function. Similar to (4), we can also derive the output values $\hat{\mathbf{y}}_{2}$ and $\hat{\mathbf{y}}_{3}$ for $f_{2}$ and $f_{3}$, respectively. For the decision-level fusion $D$, we adopt a weighted summation method

$$
\mathbf{O}=D\left(\hat{\mathbf{y}}_{1}, \hat{\mathbf{y}}_{2}, \hat{\mathbf{y}}_{3} ; \mathbf{U}\right)=\mathbf{u}_{1} \odot \hat{\mathbf{y}}_{1}+\mathbf{u}_{2} \odot \hat{\mathbf{y}}_{2}+\mathbf{u}_{3} \odot \hat{\mathbf{y}}_{3}
$$

where $\odot$ is an element-wise product operator, $\mathbf{u}_{1}, \mathbf{u}_{2}$ and $\mathbf{u}_{3}$ are three column vectors of $\mathbf{U}$, and the $i$ th element of $\mathbf{u}_{j}, j \in$ $\{1,2,3\}$ depends on the $i$ th class accuracy acquired by the $j$ th output layer on the training data.

\section{Network Training and Testing}

The whole network in Fig. 1 is trained in an end-toend manner using a given training set $\left\{\left(\boldsymbol{x}_{h}^{(i)}, \boldsymbol{x}_{l}^{(i)}, \mathbf{y}^{(i)}\right) \mid i=\right.$ $1,2, \cdots, N\}$, where $N$ represents the number of training samples, and $\mathbf{y}^{(i)}$ is the groundtruth for the $i$ th sample. After a feed-forward process, we are able to obtain three outputs for each sample. Their loss values can be computed by a crossentropy loss function. For instance, the loss value between the first output $\hat{\mathbf{y}}_{1}$ and the groundtruth $\mathbf{y}$ can be formulated as

$$
L_{1}=-\frac{1}{N} \sum_{i=1}^{N}\left[\mathbf{y}^{(i)} \log \left(\hat{\mathbf{y}}_{1}^{(i)}\right)+\left(1-\mathbf{y}^{(i)}\right) \log \left(1-\hat{\mathbf{y}}_{1}^{(i)}\right)\right] .
$$

Similarly, we can also derive $L_{2}$ and $L_{3}$ for the other two outputs. $L_{3}$ is designed to supervise the learning process of the fused feature between hyperspectral and LiDAR data, whereas $L_{1}$ and $L_{2}$ are responsible for the hyperspectral and LiDAR features, respectively. The final loss value $L$ is represented as the combination of $L_{1}, L_{2}$, and $L_{3}$

$$
L=\lambda_{1} L_{1}+\lambda_{2} L_{2}+L_{3}
$$

where $\lambda_{1}$ and $\lambda_{2}$ represent the weight parameters for $L_{1}$ and $L_{2}$, respectively. In the experiments, we empirically set them to 0.01 because it can achieve satisfactory performance. The effects of them on the classification performance will be analyzed in Section III-D. 
TABLE I

Numbers of TRAINING AND TEST SAMPLES IN EACH Class FOR THE HOUSTON DATA

\begin{tabular}{cccc}
\hline Class No. & Class Name & Training & Test \\
\hline \hline 1 & Healthy grass & 198 & 1053 \\
2 & Stressed grass & 190 & 1064 \\
3 & Synthetic grass & 192 & 505 \\
4 & Tree & 188 & 1056 \\
5 & Soil & 186 & 1056 \\
6 & Water & 182 & 143 \\
7 & Residential & 196 & 1072 \\
8 & Commercial & 191 & 1053 \\
9 & Road & 193 & 1059 \\
10 & Highway & 191 & 1036 \\
11 & Railway & 181 & 1054 \\
12 & Parking lot 1 & 192 & 1041 \\
13 & Parking lot 2 & 184 & 285 \\
14 & Tennis court & 181 & 247 \\
15 & Running track & 187 & 473 \\
\hline \hline- & Total & 2832 & 12197 \\
\hline \multicolumn{4}{c}{}
\end{tabular}

TABLE II

Numbers of TRAining AND TeSt SAMPles in EACH Class FOR THE TRENTO DATA

\begin{tabular}{cccc}
\hline Class No. & Class Name & Training & Test \\
\hline \hline 1 & Apple trees & 129 & 3905 \\
2 & Buildings & 125 & 2778 \\
3 & Ground & 105 & 374 \\
4 & Wood & 154 & 8969 \\
5 & Vineyard & 184 & 10317 \\
6 & Roads & 122 & 3252 \\
\hline \hline- & Total & 819 & 29595 \\
\hline
\end{tabular}

The same as most CNN models, $L$ can be optimized using a backpropagation algorithm. Note that $L_{1}$ and $L_{2}$ can also be considered as regularization terms for $L_{3}$, thus reducing the overfitting risk during the network training process.

Once the network is trained, we can use it to predict the label of each test sample. First, $\mathbf{u}_{j}, j \in\{1,2,3\}$ is computed on the training set. Its $i$ th element $\mathbf{u}_{j i}$ can be derived as

$$
\begin{aligned}
\mathbf{a}_{j i} & =\frac{\sum_{\ell=1}^{N} \sum_{\mathbf{y}^{(\ell)}=i} \mathbf{I}\left(\hat{\mathbf{y}}_{j}^{(\ell)}=\mathbf{y}^{(\ell)}\right)}{\sum_{\ell=1}^{N} \mathbf{I}\left(\mathbf{y}^{(\ell)}=i\right)} \\
\mathbf{u}_{j i} & =\frac{\mathbf{a}_{j i}+10^{-5}}{\mathbf{a}_{1 i}+\mathbf{a}_{2 i}+\mathbf{a}_{3 i}+10^{-5}}
\end{aligned}
$$

where $\mathbf{a}_{j i}$ is the $i$ th class accuracy of the $j$ th output, and $\mathbf{I}$ is an indicator function, the value of which equals 1 when the condition exists and 0 otherwise. Second, for the $t$ th test sample, we are able to obtain three output values $\hat{\mathbf{y}}_{1}^{(t)}, \hat{\mathbf{y}}_{2}^{(t)}$, and $\hat{\mathbf{y}}_{3}^{(t)}$ via a feed-forward propagation. Finally, the output value can be derived by using (5).

\section{EXPERIMENTS}

\section{A. Data Description}

We test the effectiveness of our proposed model on two hyperspectral and LiDAR fusion data sets.

1) Houston Data: The first data were acquired over the University of Houston campus and the neighboring urban area in June 2012 [8]. It consists of a hyperspectral image

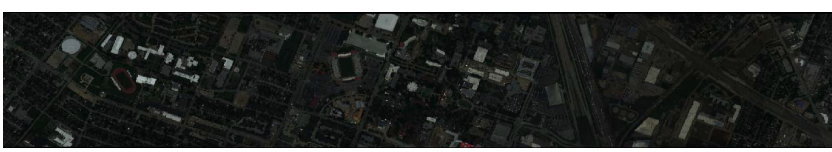

(a)

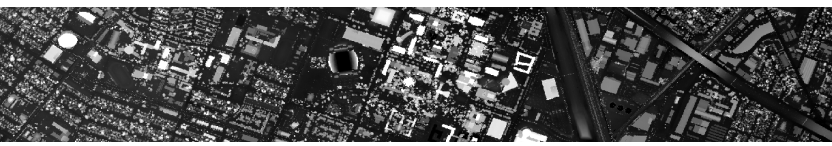

(b)

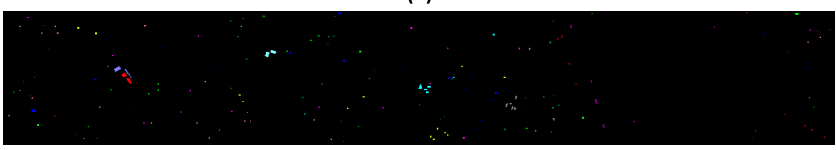

(c)

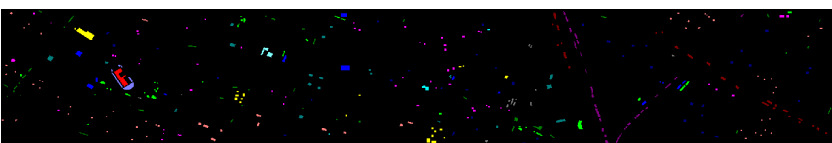

(d)

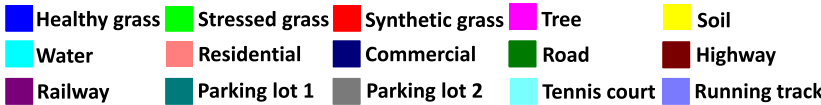

Fig. 4. Visualization of the Houston data. (a) Pseudo-color image for the hyperspectral data using 64, 43, and 22 as R, G, B, respectively. (b) Grayscale image for the LiDAR data, (c) Training data map. (d) Test data map.

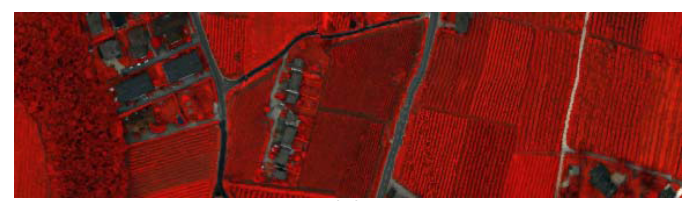

(a)

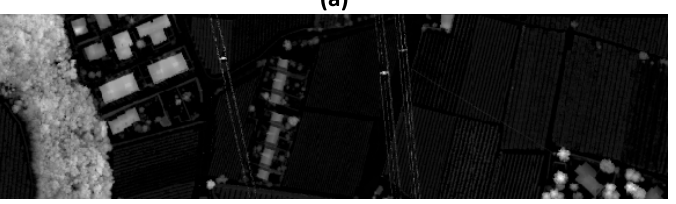

(b)

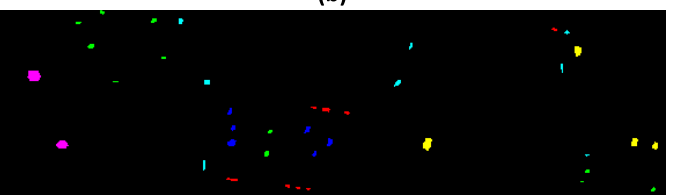

(c)

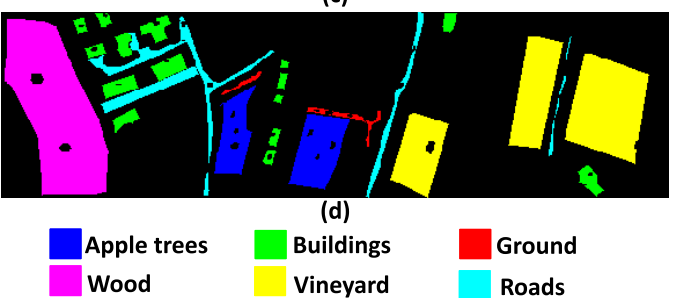

Fig. 5. Visualization of the Trento data. (a) Pseudo-color image for the hyperspectral data using 40, 20, and 10 as R, G, B, respectively. (b) Grayscale image for the LiDAR data. (c) Training data map. (d) Test data map.

and LiDAR data, both of which contain $349 \times 1905$ pixels with a spatial resolution of $2.5 \mathrm{~m}$. The number of spectral bands for the hyperspectral data is 144. Fig. 4 demonstrates a pseudocolor image of the hyperspectral data, a grayscale image of the LiDAR data, and groundtruth maps of the training and test samples. As shown in the figure, there exist 15 different 
classes. The detailed numbers of samples for each class are reported in Table I. It is worth noting that we use the standard sets of training and test samples which makes our results fully comparable with several works such as [7] and [8].

2) Trento Data: The second data were captured over a rural area in the south of Trento, Italy. The LiDAR data was acquired by the Optech ALTM 3100EA sensor, and the hyperspectral data was acquired by the AISA Eagle sensor with 63 spectral bands. The size of these two data is $166 \times 600$ pixels, and the spatial resolution is $1 \mathrm{~m}$. Fig. 5 visualizes this data, and Table II lists the number of samples in six different classes. Again, we also use the standard sets of training and test samples to construct experiments.

\section{B. Experimental Setup}

In order to validate the effectiveness of our proposed models, we comprehensively compare it with several different models. Specifically, we first select the HS network (i.e., CNNHS) and the LiDAR network (i.e., CNN-LiDAR) in Fig. 1 as two baselines and compare different fusion methods on both Houston and Trento data. Then, we focus on the Houston data and compare our model with numerous state-of-the-art models.

All of the deep learning models are implemented in the PyTorch framework. To optimize them, we use the Adam algorithm. The batch size, the learning rate, and the number of training epochs are set to $64,0.001$, and 200 , respectively. The experiments are implemented on a personal computer with an Intel core i7-4790, 3.60-GHz processor, 32-GB RAM, and a GTX TITAN X graphic card.

The classification performance of each model is evaluated by the OA, the average accuracy (AA), the per-class accuracy, and the Kappa coefficient. OA defines the ratio between the number of correctly classified pixels to the total number of pixels in the test set, AA refers to the average of accuracies in all classes, and Kappa is the percentage of agreement corrected by the number of agreements that would be expected purely by chance.

\section{Experimental Results}

1) Comparison With Different Fusion Models: In addition to two single-source models (i.e., CNN-HS and CNN-LiDAR), we also test the effectiveness of feature-level fusion models, i.e., using $f_{3}$ only. The three feature-level fusion methods CNN-F-C, CNN-F-M, and CNN-F-S stand for the concatenation method, the maximization method, and the summation method, respectively. Similarly, the three decision-level and feature-level fusion methods in Fig. 3 are abbreviated as CNN-DF-C, CNN-DF-M, and CNN-DF-S, respectively. Table III shows the detailed classification results of eight models on the Houston data. Several conclusions can be observed from it. First, for the single-source models, CNNHS achieves significantly better results than CNN-LiDAR in each class. It indicates that the spectral-spatial information in the hyperspectral data is more discriminative than the elevation information in the LiDAR data. Second, all of the three feature-level fusion models (i.e., CNN-F-C, CNN-F-M, and (NN-F-S) obtain higher accuracies than the CNN-HS model in most classes. This can be explained by the fact that LiDAR data can provide complementary information for the hyperspectral data, and by combining them together in a proper way, the classification performance can be improved. Third, based on the feature-level fusion models, if we further use the decision-level fusion (i.e., CNN-DF-C, CNN-DF-M, and CNN-DF-S), the performance is improved again. Taking the summation fusion method as an example, by the simultaneous use of feature-level and decision-level fusions, the $\mathrm{OA}$ is increased from $94.49 \%$ to $96.03 \%$, which is the best result ever reported in the literature. Last but not the least, compared to the widely used concatenation method, our proposed maximization and summation fusion methods can achieve better OA, AA, and Kappa values. Besides the quantitative results, we also qualitatively analyze the performance of different models. Fig. 6 demonstrates the classification maps of different models. In this figure, different colors represent different classes of objects. From Fig. 6(b), we can see that the CNNLiDAR model generates many outliers, and misclassifies a lot of objects. In comparison with it, other models obtain more homogeneous classification maps. However, some objects are a little over-smoothed because all of the models use the small patches and cubes as inputs.

Similar to the Houston data, Table IV and Fig. 7 show the quantitative and qualitative results, respectively, on the Trento data. The data have larger and more homogeneous objects to discriminate than the Houston data, so all of the models can achieve relatively high performance (e.g., the OA values are larger than 90\%). Specifically, CNN-HS is better than CNN-LiDAR, and the feature-level fusion method can improve the performance of CNN-HS. More importantly, simultaneous feature-level and decision-level fusion is more effective than using feature-level fusion only. The best results appear when adopting the maximization fusion method.

2) Comparison With State-of-the-Art Models: In the existing hyperspectral and LiDAR data fusion works, most of the models tested their performance on the Houston data. To highlight the superiority of our proposed models, we also compared them with state-of-the-art models, including seven traditional models and five CNN-related models, using standard training and test sets. These traditional models include the multiple feature learning model MLR $R_{\text {sub }}$ in [38], the generalized graphbased fusion model GGF in [11], the sparse and low-rank component analysis model SLRCA in [12], the total variation component analysis model OTVCA in [13], the adaptive differential evolution-based fusion model ODF-ADE in [19], the unsupervised graph fusion model E-UGF in [20], and the composite kernel extreme learning machine model HyMCKs in [39]. The CNN-related models include the deep fusion model DF in [30], the CNN model combined with graphbased feature fusion method CNNGBFF in [28], the threestream CNN-based composite kernel model CNNCK in [29], the two-branch CNN model TCNN in [31], and the patch-topatch CNN model PToPCNN in [32].

Table $\mathrm{V}$ reports the detailed comparison results of different models in terms of OA, AA, and Kappa coefficients. Note that all the results are directly cited from their original articles because we are not able to reproduce them due to missing 
TABLE III

Classification Accuracies (\%) and Kappa Coefficients of Different Models on the Houston Data. The Best Accuracies ARE SHOWN With THE Bold TyPE FACE

\begin{tabular}{|c|c|c|c|c|c|c|c|c|}
\hline Class No. & CNN-HS & CNN-LiDAR & CNN-F-C & CNN-F-M & CNN-F-S & CNN-DF-C & CNN-DF-M & CNN-DF-S \\
\hline 1 & 82.91 & 60.30 & 82.91 & 81.86 & 89.93 & 82.81 & 83.00 & 85.57 \\
\hline 2 & 99.91 & 24.34 & 99.81 & 99.44 & 98.21 & 100 & 99.81 & 99.81 \\
\hline 3 & 91.29 & 66.53 & 97.43 & 97.03 & 98.61 & 96.44 & 97.62 & 97.62 \\
\hline 4 & 95.93 & 88.73 & 99.43 & 99.05 & 99.05 & 98.96 & 99.91 & 99.43 \\
\hline 5 & 100 & 24.81 & 100 & 98.86 & 99.72 & 100 & 99.91 & 100 \\
\hline 6 & 93.71 & 25.87 & 96.50 & 100 & 100 & 100 & 100 & 95.80 \\
\hline 7 & 91.60 & 61.19 & 87.41 & 96.74 & 91.98 & 91.32 & 90.39 & 95.24 \\
\hline 8 & 87.18 & 84.33 & 91.17 & 92.69 & 96.30 & 92.40 & 95.54 & 96.39 \\
\hline 9 & 86.87 & 40.32 & 87.25 & 92.92 & 92.92 & 89.33 & 93.86 & 93.20 \\
\hline 10 & 97.59 & 53.86 & 98.75 & 84.94 & 88.51 & 99.71 & 96.04 & 98.84 \\
\hline 11 & 89.56 & 80.46 & 97.15 & 97.34 & 96.49 & 99.43 & 98.39 & 96.77 \\
\hline 12 & 91.16 & 29.30 & 96.25 & 92.22 & 86.65 & 92.51 & 93.18 & 92.60 \\
\hline 13 & 88.77 & 81.05 & 92.98 & 92.63 & 89.82 & 89.82 & 92.98 & 92.98 \\
\hline 14 & 89.07 & 52.63 & 93.52 & 100 & 99.60 & 88.26 & 95.95 & 99.19 \\
\hline 15 & 90.91 & 29.81 & 100 & 92.81 & 99.58 & 100 & 98.73 & 100 \\
\hline $\mathrm{OA}$ & 92.05 & 54.52 & 94.37 & 93.92 & 94.49 & 94.74 & 95.29 & 96.03 \\
\hline AA & 91.76 & 53.57 & 94.70 & 94.57 & 95.16 & 94.73 & 95.69 & 96.23 \\
\hline Kappa & 0.9136 & 0.5082 & 0.9389 & 0.9340 & 0.9402 & 0.9429 & 0.9488 & 0.9569 \\
\hline
\end{tabular}

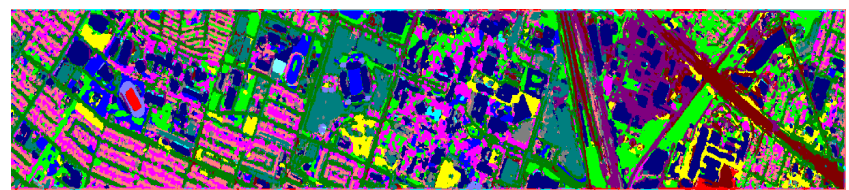

(a)

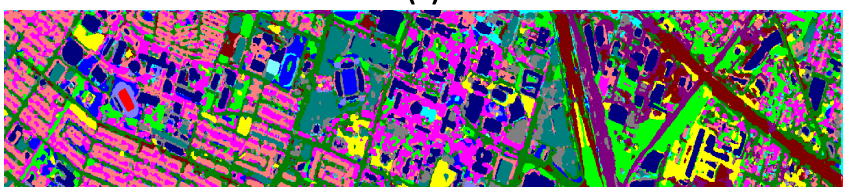

(c)

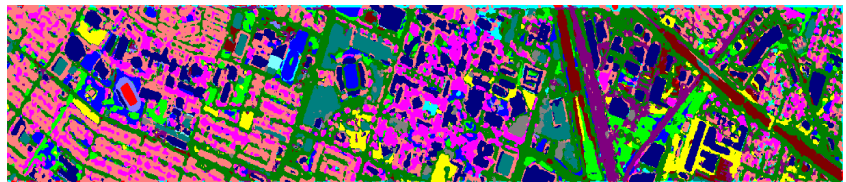

(e)

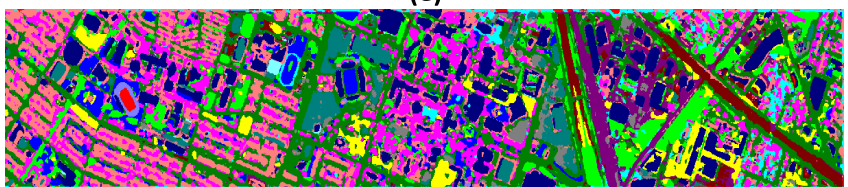

(g)

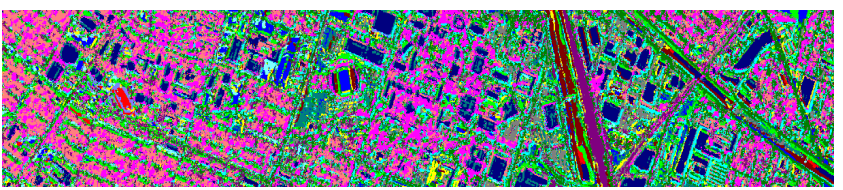

(b)

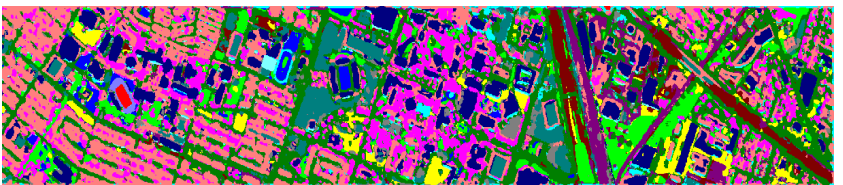

(d)

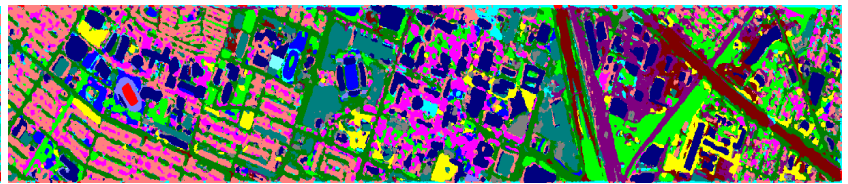

(f)

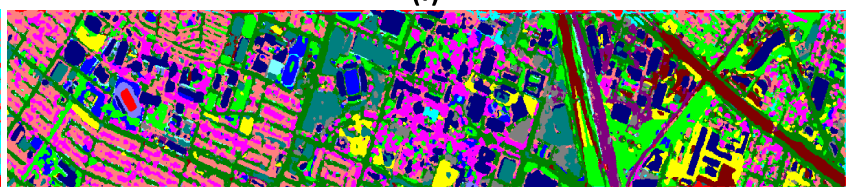

(h)

Fig. 6. Classification maps of the Houston data using different models. (a) CNN-HS. (b) CNN-LiDAR. (c) CNN-F-C. (d) CNN-F-M. (e) CNN-F-S (f) CNN-DF-C. (g) CNN-DF-M. (h) CNN-DF-S.

parameters or the availability of codes. For the traditional models, the best OA, AA, and Kappa values are $95.11 \%, 94.57 \%$, and 0.9447 , respectively, achieved by a recent work named EUGF [20]. For the CNN-related models, CNNCK [29] obtains the best OA and Kappa values, while PToPCNN [32] acquires the best AA. Compared to the E-UGF model, both CNNCK and PToPCNN models obtain inferior performance, which indicate that the existing $\mathrm{CNN}$-related fusion models still have some potentials to explore. Similar to DF [30] and TCNN [31] models, our proposed models (i.e., CNN-DF-M and CNNDF-S) can also be considered as a two-branch CNN model.
However, the proposed models can obtain significantly better results than them, even than E-UGF, which sufficiently certify the effectiveness of the proposed model.

\section{Analysis on the Proposed Model}

1) Analysis on the Reduced Dimensionality: For the proposed model, we have two hyperparameters to predefine. The first one is the number of reduced dimensionality $k$ of hyperspectral data using PCA, and the second one is the neighboring size $p \times p$ extracted from hyperspectral and 
TABLE IV

Classification Accuracies (\%) and Kappa Coefficients of Different Models on the Trento Data. The Best Accuracies ARE SHOWn With THE Bold Type FACE

\begin{tabular}{ccccccccc}
\hline Class No. & CNN-HS & CNN-LiDAR & CNN-F-C & CNN-F-M & CNN-F-S & CNN-DF-C & CNN-DF-M & CNN-DF-S \\
\hline \hline 1 & 99.85 & 99.92 & 98.49 & 96.72 & 99.15 & 98.44 & 99.69 & 99.64 \\
2 & 94.67 & 93.16 & 97.01 & 97.05 & 96.36 & 97.73 & 98.81 & 97.66 \\
3 & 82.09 & 60.43 & 92.51 & 95.99 & 93.05 & 88.50 & 94.39 & 92.25 \\
4 & 98.73 & 99.12 & 99.11 & 100 & 100 & 100 & 99.88 & 99.96 \\
5 & 99.73 & 95.63 & 100 & 100 & 99.96 & 100 & 100 & 99.90 \\
6 & 76.31 & 50.59 & 90.53 & 92.69 & 89.71 & 93.64 & 94.00 & 92.40 \\
\hline \hline OA & 96.31 & 91.91 & 98.17 & 98.48 & 98.37 & 98.77 & $\mathbf{9 9 . 1 2}$ & 98.80 \\
AA & 91.90 & 83.14 & 96.28 & 97.08 & 96.37 & 96.39 & $\mathbf{9 7 . 8 0}$ & 96.97 \\
Kappa & 0.9505 & 0.8917 & 0.9754 & 0.9796 & 0.9782 & 0.9835 & $\mathbf{0 . 9 8 8 1}$ & 0.9839 \\
\hline
\end{tabular}

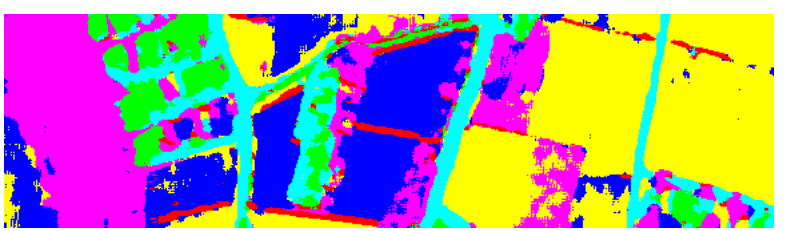

(a)

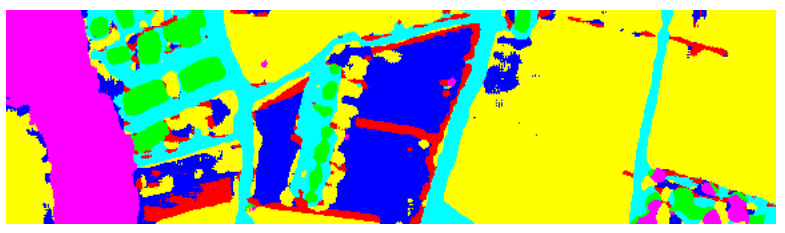

(c)

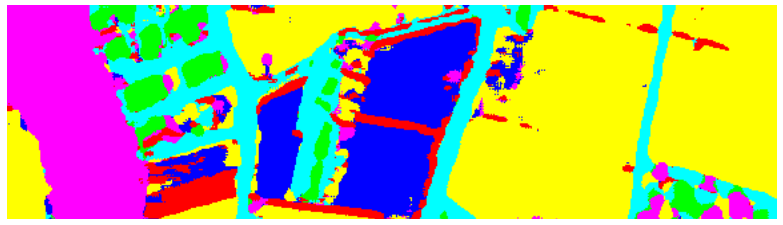

(e)

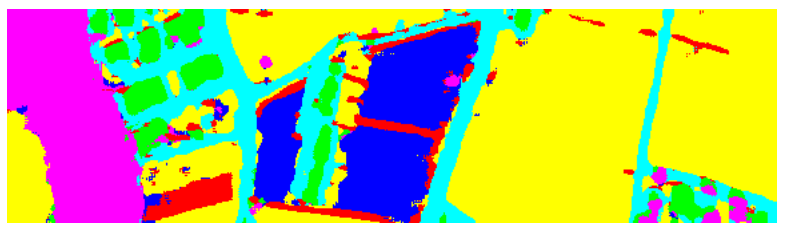

(g)

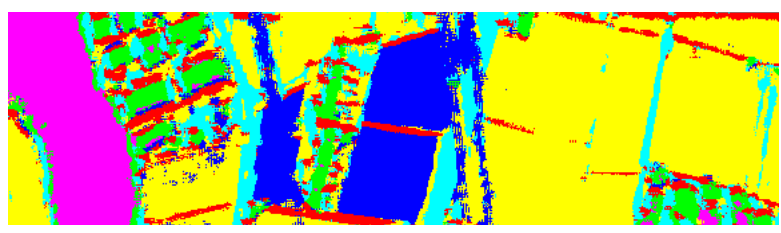

(b)

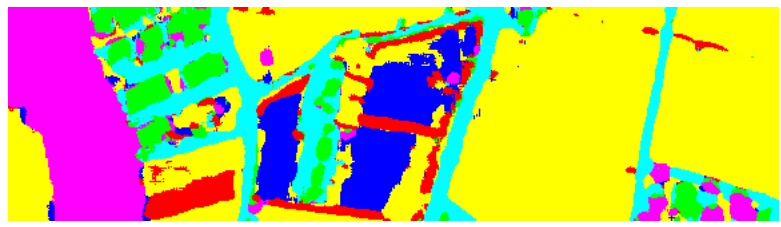

(d)

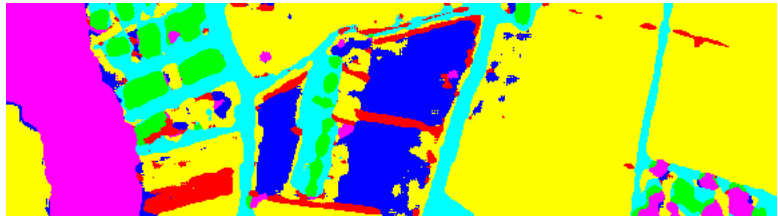

(f)

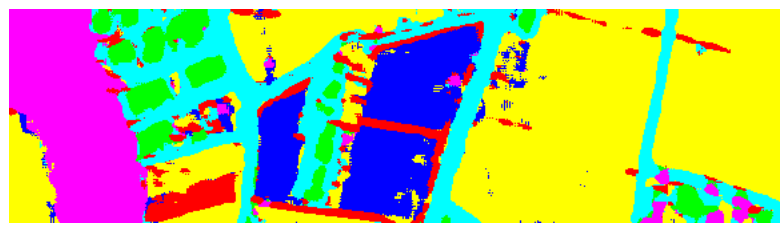

(h)

Fig. 7. Classification maps of the Trento data using different models. (a) CNN-HS. (b) CNN-LiDAR. (c) CNN-F-C. (d) CNN-F-M. (e) CNN-F-S. (f) CNNDF-C. (g) CNN-DF-M. (h) CNN-DF-S.

LiDAR data. To evaluate the effect of $k$, we fix $p$ and select $k$ from a candidate set $\{1,5,10,15,20,25,30\}$. Since the fusion models have the same hyperparameter values as single models (i.e., CNN-HS and LiDAR-HS), we only demonstrate the results of single models here. Fig. 8 shows the performance (i.e., OA) of CNN-HS on the Houston (the blue line) and Trento (the red line) data. From this figure, we can observe that as $k$ increases, OA firstly increases and then tends to a stable state. Considering the computation complexity and classification performance, $k$ can be set to 20 for both data.

2) Analysis on the Neighboring Size: Similar to the analysis of $k$, we can also fix $k$ and choose $p$ from a candidate set $\{9,11,13,15,17,19\}$ to evaluate the effect of $p$. Table VI reports the changes in $\mathrm{OA}$ values at different sizes. When the size increases from 9 to 11 on the Houston data, the improvements of OA acquired by CNN-HS and CNN-LiDAR are more than $1 \%$. But for the other sizes, these two models do not change significantly. For the Trento data, CNN-HS is relatively stable when the size changes, but CNN-LiDAR will increase more than $1 \%$ from 9 to 11 , and decrease from 11 to 13 . Based on the above analysis, 11 is a reasonable choice for CNN-HS and CNN-LiDAR on both data. This choice is consistent with the works in [30] and [32].

3) Analysis on the Coupling Strategy: Benefiting from the coupling strategy, the number of parameters in the second and the third convolutional layers is reduced by two times. Taking CNN-DF-M and CNN-DF-S models as examples, on the Houston data, the total number of parameters to train is 
TABLE V

Performance Comparison With the State-OF-The-Art Models on the Houston Data

\begin{tabular}{cccccccc}
\hline \multicolumn{7}{c}{ Traditional models } \\
\hline Model & MLR $_{\text {sub }}$ & GGF & SLRCA & OTVCA & ODF-ADE & E-UGF & HyMCKs \\
OA & 92.05 & 94.00 & 91.30 & 92.45 & 93.50 & 95.11 & 90.33 \\
AA & 92.87 & 93.79 & 91.95 & 92.68 & - & 94.57 & 91.14 \\
Kappa & 0.9137 & 0.9350 & 0.9056 & 0.9181 & 0.9299 & 0.9447 & 0.8949 \\
\hline \hline \multicolumn{7}{c}{ CNN-related models } \\
\hline Model & DF & CNNGBFF & CNNCK & TCNN & PToPCNN & CNN-DF-M & CNN-DF-S \\
OA & 91.32 & 91.02 & 92.57 & 87.98 & 92.48 & $\mathbf{9 5 . 2 9}$ & $\mathbf{9 6 . 0 3}$ \\
AA & 91.96 & 91.82 & 92.48 & 90.11 & 93.55 & $\mathbf{9 5 . 6 9}$ & $\mathbf{9 6 . 2 3}$ \\
Kappa & 0.9057 & 0.9033 & 0.9193 & 0.8698 & 0.9187 & $\mathbf{0 . 9 4 8 8}$ & $\mathbf{0 . 9 5 6 9}$ \\
\hline
\end{tabular}

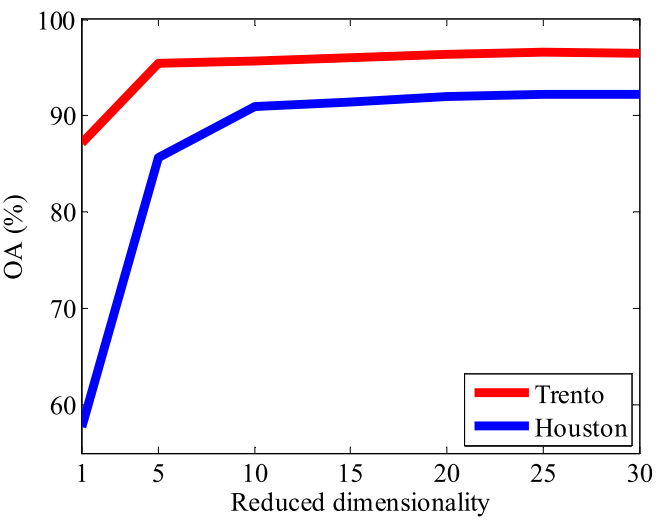

Fig. 8. Effect of the reduced dimensionality on the OA (\%) achieved by the CNN-HS model.

TABLE VI

Effect of the Neighboring Size on the OA (\%) ACQuired by the CNN-HS AND CNN-LIDAR MODELS

\begin{tabular}{ccccccc}
\hline \multicolumn{7}{c}{ Houston Data } \\
\hline Size & 9 & 11 & 13 & 15 & 17 & 19 \\
CNN-HS & 90.88 & 92.05 & 91.49 & 91.41 & 91.87 & 92.06 \\
CNN-LiDAR & 52.45 & 54.52 & 54.44 & 54.59 & 54.29 & 54.51 \\
\hline \hline \multicolumn{7}{c}{ Trento Data } \\
\hline Size & 9 & 11 & 13 & 15 & 17 & 19 \\
CNN-HS & 96.02 & 96.43 & 96.39 & 96.17 & 95.97 & 95.53 \\
CNN-LiDAR & 90.80 & 91.91 & 90.29 & 90.70 & 91.40 & 90.57 \\
\hline
\end{tabular}

TABLE VII

Computation Time (Seconds) of Different Models on the HousTON DATA

\begin{tabular}{ccccc}
\hline Time & CNN-HS & CNN-LiDAR & CNN-F-C & CNN-F-M \\
Train & 43.68 & 38.04 & 71.57 & 70.85 \\
Test & 1.24 & 1.18 & 1.30 & 1.27 \\
\hline \hline Time & CNN-F-S & CNN-DF-C & CNN-DF-M & CNN-DF-S \\
Train & 70.90 & 185.71 & 182.54 & 184.43 \\
Test & 1.28 & 1.38 & 1.33 & 1.37 \\
\hline
\end{tabular}

196128 without weight sharing, while this number is reduced to 103968 after adopting the coupling strategy; on the Trento data, the trainable parameters are 192672 and 100512 without and with weight sharing, respectively. In summary, the parameter numbers in CNN-DF-M and CNN-DF-S models are reduced by about $47 \%$ on both data when the coupling strategy
TABLE VIII

Computation Time (Seconds) of Different Models on the Trento DATA

\begin{tabular}{ccccc}
\hline Time & CNN-HS & CNN-LiDAR & CNN-F-C & CNN-F-M \\
Train & 32.11 & 21.84 & 49.99 & 49.53 \\
Test & 1.33 & 1.24 & 1.44 & 1.37 \\
\hline \hline Time & CNN-F-S & CNN-DF-C & CNN-DF-M & CNN-DF-S \\
Train & 49.62 & 118.65 & 116.43 & 117.29 \\
Test & 1.43 & 1.66 & 1.62 & 1.65
\end{tabular}
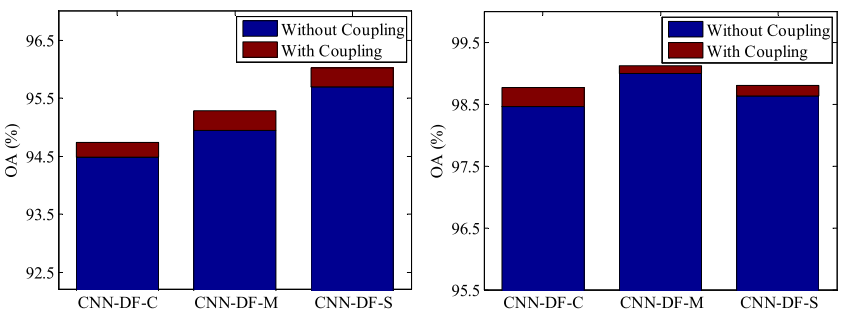

Fig. 9. Comparisons before and after adopting the coupling strategy on two data. (From left to right) Houston data and the Trento data.

is employed. Besides, we also test the effects of the coupling strategy on the classification performance. Fig. 9 illustrates the changes of OA before and after adopting the coupling strategy on the Houston data (left one) and the Trento data (right one). This indicates that the performance of CNN-DF-C, CNN-DF$\mathrm{M}$, and CNN-DF-S in terms of OA is slightly improved after adopting the coupling strategy.

4) Analysis on the Computation Cost: To quantitatively analyze the computation cost of different models, Tables VII and VIII report their computation time on the Houston and Trento data, respectively. From these two tables, we can observe that CNN-HS and CNN-LiDAR models take less training time than the other fusion models because they only need to process single-source data, without any interactions between different sources. On the contrary, the proposed decision-level and feature-level fusion models cost much more training time than the single-source and the feature-level fusion models. Nevertheless, once the networks are trained, their test efficiency is very high. In particular, it takes not more than $2 \mathrm{~s}$ to finish the test process, which is close to the time costs of the other models.

5) Analysis on the Weight Parameters: The loss function of the proposed model in (7) contains two hyper-parameters 

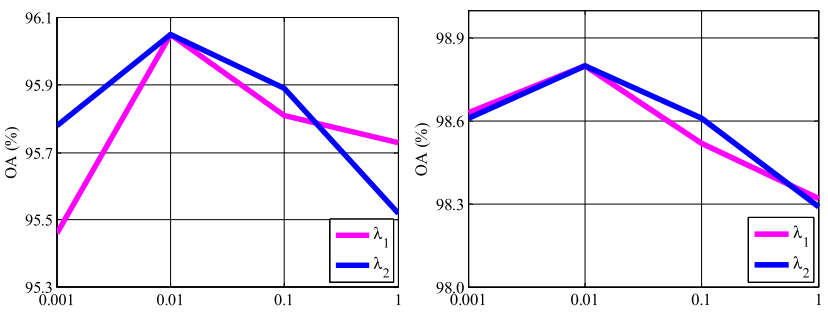

Fig. 10. Effects of weight parameters $\lambda_{1}$ and $\lambda_{2}$ on the classification performance achieved by the CNN-DF-S model on two data. (From left to right) Houston data and the Trento data.

(i.e., $\lambda_{1}$ and $\lambda_{2}$ ). In order to test their effects on the classification performance, we firstly fix $\lambda_{1}$ and change $\lambda_{2}$ from a candidate set $\{0.001,0.01,0.1,1\}$. Then, we set $\lambda_{2}$ to the optimal value and change $\lambda_{1}$ from the same set $\{0.001,0.01,0.1,1\}$. Fig. 10 shows the OAs obtained by the proposed CNN-DF-S model on the Houston and Trento data with different $\lambda_{1}$ and $\lambda_{2}$ values. In this figure, the pink and the blue lines represent the CNN-DF-S model with different $\lambda_{1}$ and $\lambda_{2}$ values, respectively. It is shown that as $\lambda_{2}$ increases, the OA will firstly increase and then decrease on both data. The highest OA value appears when $\lambda_{2}=0.01$. Similar conclusions can be observed for $\lambda_{1}$. Therefore, the optimal values for $\lambda_{1}$ and $\lambda_{2}$ are 0.01 .

\section{CONCLUSION}

This article proposed a coupled CNN framework for hyperspectral and LiDAR data fusion. Small convolution kernels and parameter sharing layers were designed to make the model more efficient and effective. In the fusion phase, we used feature-level and decision-level fusion strategies simultaneously. For the feature-level fusion, we proposed summation and maximization methods in addition to the widely used concatenation method. For the decision-level fusion, we proposed a weighted summation method, whose weights depend on the performance of each output layer. To validate the effectiveness of the proposed model, we constructed several experiments on two data sets. The experimental results show that the proposed model can achieve the best performance on the Houston data and very high performance on the Trento data. Additionally, we also thoroughly evaluated the effects of different hyperparameters on the classification performance, including the reduced dimensionality and the neighboring size. In the future, more powerful neighboring extraction methods need to be explored, because the current classification maps still exist over-smoothing problems.

\section{REFERENCES}

[1] R. Hang, Q. Liu, H. Song, and Y. Sun, "Matrix-based discriminant subspace ensemble for hyperspectral image spatial-spectral feature fusion," IEEE Trans. Geosci. Remote Sens., vol. 54, no. 2, pp. 783-794, Sep. 2015.

[2] P. Ghamisi et al., "Advances in hyperspectral image and signal processing: A comprehensive overview of the state of the art," IEEE Geosci. Remote Sens. Mag., vol. 5, no. 4, pp. 37-78, Dec. 2017.

[3] D. Hong, N. Yokoya, J. Chanussot, and X. X. Zhu, "An augmented linear mixing model to address spectral variability for hyperspectral unmixing," IEEE Trans. Image Process., vol. 28, no. 4, pp. 1923-1938, Apr. 2019.
[4] D. Hong, N. Yokoya, J. Chanussot, and X. X. Zhu, "CoSpace: Common subspace learning from hyperspectral-multispectral correspondences," IEEE Trans. Geosci. Remote Sens., vol. 57, no. 7, pp. 4349-4359, Jul. 2019.

[5] P. Ghamisi et al., "New frontiers in spectral-spatial hyperspectral image classification: The latest advances based on mathematical morphology, Markov random fields, segmentation, sparse representation, and deep learning," IEEE Geosci. Remote Sens. Mag., vol. 6, no. 3, pp. 10-43, Sep. 2018.

[6] L. He, J. Li, C. Liu, and S. Li, "Recent advances on spectral-spatial hyperspectral image classification: An overview and new guidelines," IEEE Trans. Geosci. Remote Sens., vol. 56, no. 3, pp. 1579-1597, Mar. 2018.

[7] P. Ghamisi et al., "Multisource and multitemporal data fusion in remote sensing: A comprehensive review of the state of the art," IEEE Geosci. Remote Sens. Mag., vol. 7, no. 1, pp. 6-39, Mar. 2019.

[8] C. Debes et al., "Hyperspectral and LiDAR data fusion: Outcome of the 2013 GRSS data fusion contest," IEEE J. Sel. Topics Appl. Earth Observ. Remote Sens., vol. 7, no. 6, pp. 2405-2418, Jun. 2014.

[9] M. Pedergnana, P. R. Marpu, M. Dalla Mura, J. A. Benediktsson, and L. Bruzzone, "Classification of remote sensing optical and LiDAR data using extended attribute profiles," IEEE J. Sel. Topics Signal Process., vol. 6, no. 7, pp. 856-865, Nov. 2012.

[10] Y. Zhang and S. Prasad, "Multisource geospatial data fusion via local joint sparse representation," IEEE Trans. Geosci. Remote Sens., vol. 54, no. 6, pp. 3265-3276, Jun. 2016.

[11] W. Liao, A. Pizurica, R. Bellens, S. Gautama, and W. Philips, "Generalized graph-based fusion of hyperspectral and LiDAR data using morphological features," IEEE Geosci. Remote Sens. Lett., vol. 12, no. 3 pp. 552-556, Mar. 2015.

[12] B. Rasti, P. Ghamisi, J. Plaza, and A. Plaza, "Fusion of hyperspectral and LiDAR data using sparse and low-rank component analysis," IEEE Trans. Geosci. Remote Sens., vol. 55, no. 11, pp. 6354-6365, Nov. 2017.

[13] B. Rasti, P. Ghamisi, and R. Gloaguen, "Hyperspectral and LiDAR fusion using extinction profiles and total variation component analysis," IEEE Trans. Geosci. Remote Sens., vol. 55, no. 7, pp. 3997-4007, Jul. 2017.

[14] B. Rasti, P. Ghamisi, and M. Ulfarsson, "Hyperspectral feature extraction using sparse and smooth low-rank analysis," Remote Sens., vol. 11, no. 2, p. 121, Jan. 2019.

[15] S. Niazmardi, B. Demir, L. Bruzzone, A. Safari, and S. Homayouni, "Multiple kernel learning for remote sensing image classification," IEEE Trans. Geosci. Remote Sens., vol. 56, no. 3, pp. 1425-1443, Mar. 2018.

[16] Y. Gu, Q. Wang, X. Jia, and J. A. Benediktsson, "A novel MKL model of integrating LiDAR data and MSI for urban area classification," IEEE Trans. Geosci. Remote Sens., vol. 53, no. 10, pp. 5312-5326, Oct. 2015.

[17] W. Liao, R. Bellens, A. Pizurica, S. Gautama, and W. Philips, "Combining feature fusion and decision fusion for classification of hyperspectral and LiDAR data," in Proc. IEEE Geosci. Remote Sens. Symp., Jul. 2014, pp. 1241-1244.

[18] Y. Zhang, H. L. Yang, S. Prasad, E. Pasolli, J. Jung, and M. Crawford, "Ensemble multiple kernel active learning for classification of multisource remote sensing data," IEEE J. Sel. Topics Appl. Earth Observ. Remote Sens., vol. 8, no. 2, pp. 845-858, Feb. 2015.

[19] Y. Zhong, Q. Cao, J. Zhao, A. Ma, B. Zhao, and L. Zhang, "Optimal decision fusion for urban land-use/land-cover classification based on adaptive differential evolution using hyperspectral and LiDAR data," Remote Sens., vol. 9, no. 8, p. 868, Aug. 2017.

[20] J. Xia, N. Yokoya, and A. Iwasaki, "Fusion of hyperspectral and LiDAR data with a novel ensemble classifier," IEEE Geosci. Remote Sens. Lett., vol. 15 , no. 6, pp. 957-961, Jun. 2018.

[21] L. Zhang, L. Zhang, and B. Du, "Deep learning for remote sensing data: A technical tutorial on the state of the art," IEEE Geosci. Remote Sens. Mag., vol. 4, no. 2, pp. 22-40, Jun. 2016.

[22] R. Hang, Q. Liu, D. Hong, and P. Ghamisi, "Cascaded recurrent neural networks for hyperspectral image classification," IEEE Trans. Geosci. Remote Sens., vol. 57, no. 8, pp. 5384-5394, Aug. 2019.

[23] X. X. Zhu et al., "Deep learning in remote sensing: A comprehensive review and list of resources," IEEE Geosci. Remote Sens. Mag., vol. 5, no. 4, pp. 8-36, Dec. 2017.

[24] G. Cheng, P. Zhou, and J. Han, "Learning rotation-invariant convolutional neural networks for object detection in VHR optical remote sensing images," IEEE Trans. Geosci. Remote Sens., vol. 54, no. 12, pp. 7405-7415, Dec. 2016. 
[25] Y. Chen, H. Jiang, C. Li, X. Jia, and P. Ghamisi, "Deep feature extraction and classification of hyperspectral images based on convolutional neural networks," IEEE Trans. Geosci. Remote Sens., vol. 54, no. 10, pp. 6232-6251, Oct. 2016.

[26] Q. Liu, R. Hang, H. Song, and Z. Li, "Learning multiscale deep features for high-resolution satellite image scene classification," IEEE Trans. Geosci. Remote Sens., vol. 56, no. 1, pp. 117-126, Jan. 2018.

[27] S. Morchhale, V. P. Pauca, R. J. Plemmons, and T. C. Torgersen, "Classification of pixel-level fused hyperspectral and lidar data using deep convolutional neural networks," in Proc. 8th Workshop Hyperspectral Image Signal Process., Evol. Remote Sens. (WHISPERS), Aug. 2016, pp. $1-5$.

[28] P. Ghamisi, B. Hofle, and X. X. Zhu, "Hyperspectral and LiDAR data fusion using extinction profiles and deep convolutional neural network," IEEE J. Sel. Topics Appl. Earth Observ. Remote Sens., vol. 10, no. 6, pp. 3011-3024, Jun. 2017.

[29] H. Li, P. Ghamisi, U. Soergel, and X. Zhu, "Hyperspectral and LiDAR fusion using deep three-stream convolutional neural networks," Remote Sens., vol. 10, no. 10, p. 1649, Oct. 2018.

[30] Y. Chen, C. Li, P. Ghamisi, X. Jia, and Y. Gu, "Deep fusion of remote sensing data for accurate classification," IEEE Geosci. Remote Sens. Lett., vol. 14, no. 8, pp. 1253-1257, Aug. 2017.

[31] X. Xu, W. Li, Q. Ran, Q. Du, L. Gao, and B. Zhang, "Multisource remote sensing data classification based on convolutional neural network," IEEE Trans. Geosci. Remote Sens., vol. 56, no. 2, pp. 937-949, Feb. 2018.

[32] M. Zhang, W. Li, Q. Du, L. Gao, and B. Zhang, "Feature extraction for classification of hyperspectral and LiDAR data using patch-to-patch CNN," IEEE Trans. Cybern., vol. 50, no. 1, pp. 100-111, Jan. 2020.

[33] J. Long, E. Shelhamer, and T. Darrell, "Fully convolutional networks for semantic segmentation," in Proc. IEEE Conf. Comput. Vis. Pattern Recognit. (CVPR), Jun. 2015, pp. 3431-3440.

[34] S. Yu, S. Jia, and C. Xu, "Convolutional neural networks for hyperspectral image classification," Neurocomputing, vol. 219, pp. 88-98, Jan. 2017.

[35] Y. Xu, L. Zhang, B. Du, and F. Zhang, "Spectral-spatial unified networks for hyperspectral image classification," IEEE Trans. Geosci. Remote Sens., vol. 56, no. 10, pp. 5893-5909, Oct. 2018.

[36] Z. Zhong, J. Li, Z. Luo, and M. Chapman, "Spectral-spatial residual network for hyperspectral image classification: A 3-D deep learning framework," IEEE Trans. Geosci. Remote Sens., vol. 56, no. 2 , pp. 847-858, Feb. 2018.

[37] X. He, A. Wang, P. Ghamisi, G. Li, and Y. Chen, "LiDAR data classification using spatial transformation and CNN," IEEE Geosci. Remote Sens. Lett., vol. 16, no. 1, pp. 125-129, Jan. 2019.

[38] M. Khodadadzadeh, J. Li, S. Prasad, and A. Plaza, "Fusion of hyperspectral and LiDAR remote sensing data using multiple feature learning," IEEE J. Sel. Topics Appl. Earth Observ. Remote Sens., vol. 8, no. 6 , pp. 2971-2983, Jun. 2015.

[39] P. Ghamisi, B. Rasti, and J. A. Benediktsson, "Multisensor composite kernels based on extreme learning machines," IEEE Geosci. Remote Sens. Lett., vol. 16, no. 2, pp. 196-200, Feb. 2019.

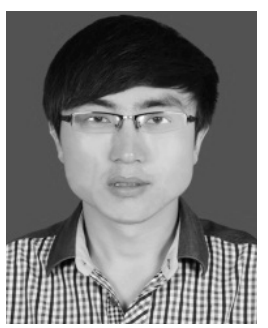

Renlong Hang (Member, IEEE) received the M.S and Ph.D. degrees from the Nanjing University of Information Science and Technology, Nanjing, China, in 2014 and 2017, respectively.

Since 2017, he has been a Lecturer with the School of Automation, Nanjing University of Information Science and Technology. From 2018 to 2019, he was a Post-Doctoral Researcher with the Department of Computer Science and Electrical Engineering, University of Missouri-Kansas City, Kansas City, MO, USA. He has authored or coauthored over 20 peerreviewed articles in international journals, such as the IEEE TRANSACTIONS on Geoscience and Remote SEnsing, the IEEE Journal of Selected TOPICS IN APPLIED EARTH ObSERVATIONS AND REMOTE SENSING, and the IEEE GeOSCIENCE AND REMOTE SENSING LetTERs. His research interests include machine learning, pattern recognition, and their applications to remote sensing image processing.

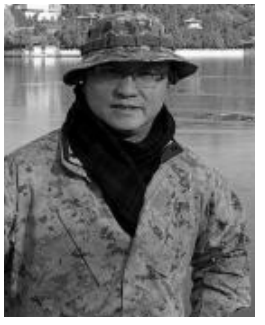

Zhu Li (Senior Member, IEEE) received the $\mathrm{Ph} . \mathrm{D}$. degree in electrical and computer engineering from Northwestern University, Evanston, IL, USA, in 2004

He was the AFRL Summer Faculty at the U.S. Air Force Academy, UAV Research Center, from 2016 to 2018. He was a Sr. Staff Researcher/Sr. Manager with Samsung Research America's Multimedia Core Standards Research Lab, Dallas, TX, USA, from 2012 to 2015, a Sr. Staff Researcher with FutureWei Media Lab, Bridgewater, NJ, USA, from 2010 to 2012, an Assistant Professor with the Department of Computing, The Hong Kong Polytechnic University, Hong Kong, from 2008 to 2010, and a Principal Staff Research Engineer with the Multimedia Research Lab (MRL), Motorola Labs, Schaumburg, IL, USA, from 2000 to 2008. He is currently an Associate Professor with the Department of CSEE, University of MissouriKansas City (UMKC), Kansas City, MO, USA, where he also directs the NSF I/UCRC Center for Big Learning. His research interests include image/video analysis, compression, and communication and associated optimization and machine learning problems. He has 46 issued or pending patents, over 100 publications in book chapters, journals, conference proceedings, and standards contributions in these areas.

Dr. Li received the Best Paper Award from the IEEE International Conference on Multimedia and Expo (ICME) at Toronto in 2006 and the Best Paper Award from the IEEE International Conference on Image Processing (ICIP) at San Antonio in 2007. He has been an Associate Editor-in-Chief (AEiC) of the IEEE TRANSACTIONS ON CIRCUITS AND SYSTEMS FOR VIDEO TECHNOLOGY since 2020 and an Associate Editor of the IEEE TRANSACTIONS ON IMAge Processing since 2019. He was an Associate Editor of the IEEE TRansactions ON Multimedia from 2015 to 2018 and the IEEE TRANSACtions on Circuits and Systems For Video TeChNOlogy from 2016 to 2019 .

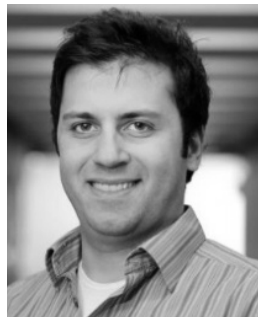

Pedram Ghamisi (Senior Member, IEEE) received the B.Sc. degree in civil (survey) engineering from the Tehran South Campus of Azad University, Tehran, Iran, and the M.Sc. degree (Hons.) in remote sensing from the K. N. Toosi University of Technology, Tehran, in 2012. In 2013/2014, he spent seven months at the School of Geography, Planning and Environmental Management, The University of Queensland, Brisbane, QLD, Australia. He received the Ph.D. degree in electrical and computer engineering from the University of Iceland, Reykjavik,

Iceland, in 2015.

After receiving his Ph.D. degree, he was a Post-Doctoral Research Fellow with the University of Iceland. In 2015, he received the prestigious Alexander von Humboldt Fellowship in 2015 and started his work as a Post-Doctoral Research Fellow with the Technical University of Munich (TUM), Munich, Germany, and Heidelberg University, Heidelberg, Germany, in October 2015. He was also a Research Scientist with the German Aerospace Center (DLR), Remote Sensing Technology Institute (IMF), Oberpfaffenhofen, Germany, from October 2015 to May 2018. In 2018, he won the prestigious High Potential Program and started his work as the Head of the Machine Learning Group, Helmholtz-Zentrum Dresden-Rossendorf (HZDR), Dresden, Germany. His research interests involve interdisciplinary research on remote sensing and machine (deep) learning, image and signal processing, and multisensory data fusion.

Dr. Ghamisi received the Best Researcher Award for M.Sc. students in K. N. Toosi University of Technology in the academic year 2010-2011. At the 2013 IEEE International Geoscience and Remote Sensing Symposium (IGARSS), Melbourne, July 2013, he received the IEEE Mikio Takagi Prize for winning the Student Paper Competition, competing with almost 70 submissions. In 2016, he was selected as the Talented International Researcher by Iran's National Elites Foundation. In 2017, he won the Data Fusion Contest 2017 organized by the Image Analysis and Data Fusion Technical Committee (IADF) of the Geoscience and Remote Sensing Society (IEEE-GRSS). He was also the Winner of the 2017 Best Reviewer Prize of the IEEE GeOsCIENCE AND REMOTE SENSING LETTERS (GRSL). He serves as an Associate Editor for the IEEE Geoscience And Remote Sensing Letters (GRSL) and Remote Sensing. 


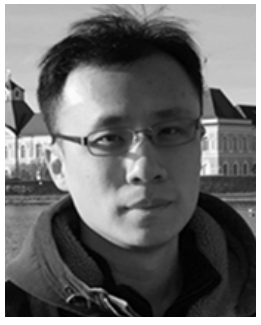

Danfeng Hong (Member, IEEE) was born in Shandong, China, in 1989. He received the B.Sc. degree in computer science and technology from the Neusoft College of Information, Northeastern University, Shenyang, China, in 2012, the M.Sc. degree (summa cum laude) in computer vision from the College of Information Engineering, Qingdao University, Qingdao, China, in 2015, and the Dr.-Ing degree (summa cum laude) from Signal Processing in Earth Observation (SiPEO), Technical University of Munich (TUM), Munich, Germany, in 2019.

Since 2015, he has been a Research Associate with the Remote Sensing Technology Institute (IMF), German Aerospace Center (DLR), Oberpfaffenhofen, Germany. He is currently a Research Scientist and leads the Spectral Vision Group, EO Data Science, IMF, DLR. From 2018 to 2019, he was a Visiting Scholar with GIPSA-lab, Grenoble INP, CNRS, Univ. Grenoble Alpes, Grenoble, France, and in RIKEN Artificial Intelligent Project (AIP), RIKEN, Tokyo, Japan. His research interests include signal/image processing and analysis, pattern recognition, machine/deep learning, and their applications in earth vision.

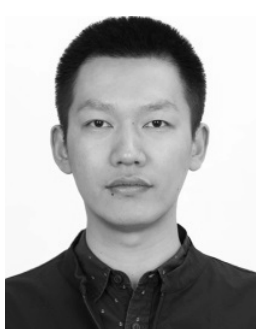

Guiyu Xia received the B.S. degree in software engineering and the Ph.D. degree in pattern recognition and intelligent system from the School of Computer Science and Engineering, Nanjing University of Science and Technology, Nanjing, China, in 2012 and 2017, respectively.

$\mathrm{He}$ is currently a Lecturer with the School of Automation, Nanjing University of Information Science and Technology, Nanjing. His research interest covers pattern recognition, machine learning, human motion analysis, and motion synthesis.

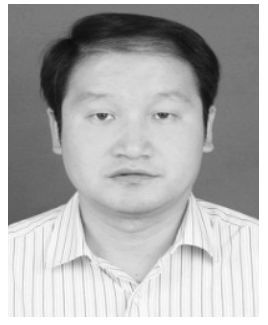

Qingshan Liu (Senior Member, IEEE) received the M.S. degree from Southeast University, Nanjing, China, in 2000, and the Ph.D. degree from the Chinese Academy of Sciences, Beijing, China, in 2003 .

From 2010 to 2011, he was an Assistant Research Professor with the Department of Computer Science, Computational Biomedicine Imaging and Modeling Center, Rutgers, The State University of New Jersey, Piscataway, NJ, USA. Before he joined Rutgers University, he was an Associate Professor with the National Laboratory of Pattern Recognition, Chinese Academy of Sciences. From June 2004 to April 2005, he was an Associate Researcher with the Multimedia Laboratory, The Chinese University of Hong Kong, Hong Kong. $\mathrm{He}$ is currently a Professor with the School of Information and Control, Nanjing University of Information Science and Technology, Nanjing. His research interests include image and vision analysis.

Dr. Liu received the President Scholarship of the Chinese Academy of Sciences in 2003. 